\title{
Panorama perceptual de la aplicación del Programa Pueblos Mágicos en Jerez, Zacatecas
}

\section{Perceptual overview of the application of the Magic Towns Program in Jerez, Zacatecas}

Nora Edith Alcalá-Flores; Mayra Janeth Alcalá-Aguilar;

Marlene Italia Botello3; Nayere Palos-Sánchez ${ }^{4}$

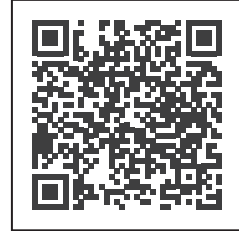

\section{Palabras clave:}

comunidad local; desarrollo económico y social; estadísticas; investigación; marca; pueblo mágico; turismo cultural.

Artículo de investigación

Fecha de recepción:

15 de marzo de 2021

Fecha de aprobación:

11 de octubre de 2021

Fecha de publicación:

22 de noviembre de 2021

Esta publicación se encuentra bajo licencia:

Creative Commons

Reconocimiento-

NoComercial-SinObraDerivada 4.0 Internacional

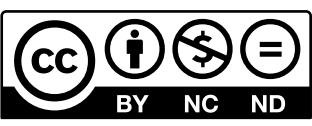

\section{Resumen}

Problemática: en 2007 Jerez se incorpora al Programa Pueblos Mágicos de la Secretaría de Turismo del Gobierno Federal, buscando un consecuente incremento de la actividad turística. Objetivos: la motivación para realizar esta investigación es la de caracterizar, desde la perspectiva y opinión de autoridades locales, habitantes, turistas y empresarios, los beneficios obtenidos, si es que estos son perceptibles para ellos. Materiales y métodos: bajo una metodología de investigación con un enfoque mixto, el estudio de campo recabó datos mediante encuestas específicamente diseñadas para cada población objeto de estudio. El tratamiento y el análisis de los datos se llevó a cabo mediante un software estadístico. Resultados y discusión: con base en el análisis de resultados se puede determinar que, de acuerdo con la percepción de los pobladores, empresarios y autoridades, no existe

1 Licenciatura en Mercadotecnia, maestría en Estadística Aplicada y doctorado en Planeación Estratégica y Dirección de Tecnología, Universidad Tecnológica del Estado de Zacatecas, RedAyN, México. nalcala@utzac.edu.mx, ORCID: https://orcid.org/0000-0002-8991-6183

2 Licenciatura en Administración de Empresas y maestría en Mercadotecnia, Universidad Tecnológica del Estado de Zacatecas, RedAyN, México. malcala@ utzac.edu.mx, ORCID: https://orcid.org/0000-0003-0518-6310

3 Licenciatura en Administración, Universidad Tecnológica del Estado de Zacatecas, RedAyN, México.mbotello@utzac.edu.mx, ORCID: https://orcid. org/0000-0003-0318-1882

4 Medicina Veterinaria y Zootecnia y maestría en Ciencias Fisiológicas, Universidad Tecnológica del Estado de Zacatecas, RedAyN, México.npalos@utzac. edu.mx, ORCID: https://orcid.org/0000-0002-4232-2767 


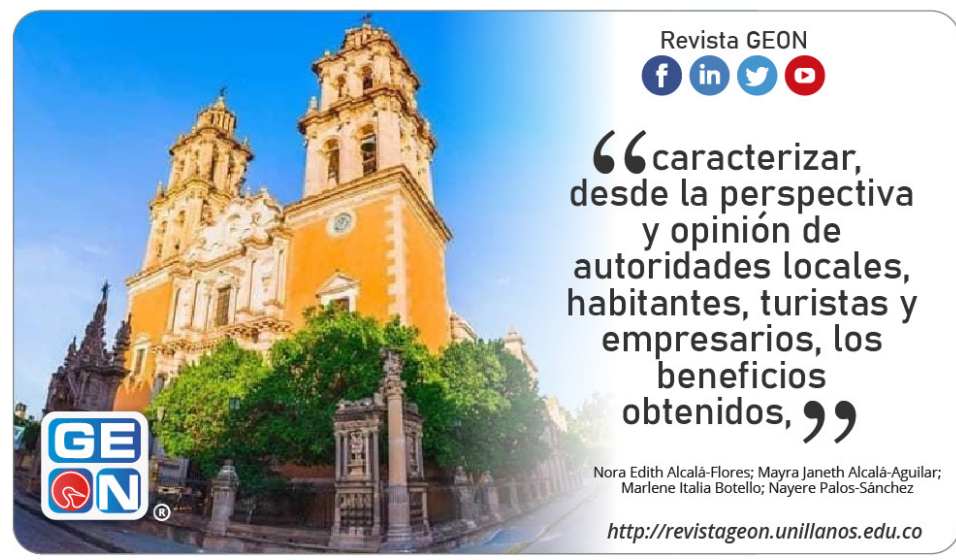

información estadística suficiente para asegurar que el Programa Pueblos Mágicos del Gobierno Federal haya impulsado el desarrollo de Jerez. Conclusiones: la investigación concluye que existen diferencias en los beneficios percibidos de acuerdo con la población de estudio que se trate, por lo que deben considerarse los aspectos en los que existen diferencias perceptivas para identificar las estrategias en las cuales se depositan los esfuerzos. Contribución: en general, se aportan estrategias que pueden contribuir a un mejor aprovechamiento de la marca de Pueblo Mágico en esa localidad y, sobre todo, incidir positivamente en su desarrollo social y económico.

Palabras clave: comunidad local; desarrollo económico y social; estadísticas; investigación; marca; pueblo mágico; turismo cultural.

Códigos JEL: Z32 Turismo y desarrollo; Z33 Marketing y finanzas

Cómo citar este artículo / Toreference this article:

Alcalá-Flores, N. E., Alcalá-Aguilar, M. J., Botello, M. I., \& Palos-Sánchez, N. (2021). Panorama perceptual de la aplicación del Programa Pueblos Mágicos en Jerez, Zacatecas. Revista GEON (Gestión, Organizaciones Y Negocios), 8(2), e-317. https://doi. org/10.22579/23463910.317

\section{Abstract}

Problematic: In 2007, Jerez joined the "Magic Towns" Program of the Tourism Department of the Federal Government, seeking a consequent increase in tourist activity; Objectives: The motivation to carry out this research is to characterize, from the perspective and opinion of local authorities, inhabitants, tourists and businesspeople, the benefits obtained; if they are perceptible to them. Mate- 
Cómo citar este artículo / Toreference this article.

Alcalá-Flores, N. E., Alcalá-Aguilar, M. J., Botello, M. I., \& Palos-Sánchez, N. (2021). Panorama perceptual de la aplicación del Programa Pueblos Mágicos en Jerez, Zacatecas. Revista GEON (Gestión, Organizaciones Y Negocios), 8(2), e-317. https://doi. org/10.22579/23463910.317 rials and methods: Under a research methodology with a mixed approach, the field study collected data through surveys specifically designed for each population under study; such data treatment and analysis were carried out using statistical software. Results and Discussion: Derived from the analysis of the results, it can be determined that, according to the perception of the residents, businesspeople and authorities, there is not enough statistical information to ensure that the Magical Towns program of the Federal Government has promoted the development of Jerez. Conclusions: The research concludes that there are differences within the perceived benefits according to the study population in question, so the aspects in which there are perceptual differences should be considered to identify the strategies in which to place efforts. Contribution: In general, strategies that contribute to a better use of the Magic Town brand in that town are provided; still, above all, would have a positive impact on its social and economic development.

Keywords: brand; cultural tourism; economic and social development; local community; statistics; research.

JEL Codes: Z32 Development and Tourism; Z33 Marketing and Finance

\section{Introducción}

Los pueblos que tienen gran influencia del pasado indígena o legado del antiguo imperio colonial español han sido seleccionados por el programa desarrollado por la Secretaría de Turismo (SECTUR), llamado Pueblos Mágicos, para llevar a cabo la tarea de conservar, proteger y guardar su riqueza cultural para las futuras generaciones. Desde el nacimiento del programa en el año 2001, hasta la fecha, se ha otorgado el nombramiento de Pueblo Mágico a un total de 123 localidades. Y cada una de ellas recibe un apoyo gubernamental y federal en forma de fondos para conservar y mejorar sus pueblos.

En esta investigación se pretende determinar el nivel de contribución del Programa Pueblos Mágicos en la localidad de Jerez de García Salinas, evaluando el impacto en la generación de empleos y en las unidades e ingresos económicos como resultado de la atracción y actividad turística. Se ana- 
liza la percepción del grado de participación y beneficio obtenido con la operación del programa; así mismo, se evalúa el desarrollo de la comunidad desde la perspectiva personal de las autoridades locales, la población, los turistas y los empresarios. A través de una adecuada metodología, se tratan a profundidad los hallazgos correspondientes a esta localidad, la cual está enclavada en el estado de Zacatecas. Finalmente, se proponen estrategias para contribuir al verdadero aprovechamiento de este Pueblo Mágico, especialmente en la industria del turismo, cuya derrama económica ya es parte vital.

\section{Contexto teórico}

De acuerdo con la Secretaría de Turismo del Gobierno Federal (2019), se atribuye el título de Pueblo Mágico a aquella localidad que cuenta con atributos simbólicos, legado histórico, hechos trascendentes, el comportamiento cotidiano de la propia población, sus tradiciones y costumbres, algo que pueda catalogarse como magia y que, a través de la visión de esta dependencia gubernamental, se convierte en una estrategia para el desarrollo del turismo en todo el país, por medio de la implementación del Programa Pueblos Mágicos.

Incorporarse a una estrategia de este tipo requiere un compromiso por parte de la localidad aspirante y del Gobierno del estado al que pertenece. Las localidades deben prepararse y contar con un inventario vigente de recursos y atractivos turísticos con evidencia fotográfica y un directorio vigente que contemple la oferta de servicios turísticos, en alineación con el Plan Nacional de Desarrollo, así como con el Programa Sectorial de Turismo, y las propias ofertas vigentes del estado. Se debe contar con el Programa Municipal de Turismo.

De acuerdo con Caro et al. (2015), ante la globalización, es importante atrapar a través de elementos de identidad de una región a quienes, en la actualidad, en lo que al turismo respecta, están interesados en sus cualidades y en recibir algo más para tomar decisiones con respecto a los sitios por visitar. Pueblos Mágicos ha sido registrado por la Secretaría de Turismo ante el Instituto Mexicano de Propiedad Industrial (IMPI) como signo distintivo y denominación, lo que implica que dicha marca pueda ir construyendo un posicionamiento, siempre que el impacto de esta sea equilibrado entre las 123 localidades que la poseen (SECTUR, 2019).

Ningún escenario puede ser plausible sin el capital humano, por lo cual se requiere tener una visión clara sobre la capacitación y profesionalización de los prestadores de servicios turísticos. La sensibilización que la administración pueda generar entre los prestadores de servicios en la búsqueda de la calidad y la certificación turística será, sin duda, uno de los detonantes a considerar en el éxito de la iniciativa para pertenecer a este programa (SECTUR, 2019).

De acuerdo con Landeros et al. (2015), los casos estudiados en su investigación mostraron que el progra- 
ma Pueblos Mágicos ha contribuido relativamente en la oferta turística, ya que se debe considerar la accesibilidad y los servicios turísticos tanto de la localidad como de los alrededores. Aparentemente, los esfuerzos principales del programa se han orientado hacia la mejora de la imagen urbana; sin embargo, no se distinguen de forma clara y, sobre todo, no se pueden medir los beneficios.

\section{Materiales y métodos}

Con base en el horizonte que se busca en la presente investigación, es importante señalar que se trabaja con un enfoque mixto (cualitativo y cuantitativo) que permita explicar la realidad del Pueblo Mágico de Jerez desde una perspectiva externa y, a su vez, objetiva. Por lo anterior, se identificó en primera instancia la población objeto de estudio y se encontró pertinente considerar la perspectiva de cuatro poblaciones involucradas en el tema de investigación para tener realmente una investigación completa y apropiada (Hernández et al., 2010). Debido a que se identifican cuatro poblaciones diferentes para el estudio planteado, no puede tratarse de una muestra única o general para el Pueblo Mágico.

Las muestras de cada población se calcularon con base en información documentada en el Instituto Nacional de Estadística y Geografía (INEGI, 2019 y 2020) y SECTUR (2019), utilizando un error de estimación del 5 $\%$ y un nivel de confianza del $95 \%$. Todas las poblaciones son finitas, tal como se ilustra en la tabla 1, la cual detalla el tamaño respectivo de la muestra para cada población.

Tabla 1.Poblaciones y muestras

\begin{tabular}{|c|c|c|c|}
\hline Población & Características & $\begin{array}{c}\text { Tamaño } \\
\text { población }\end{array}$ & $\begin{array}{l}\text { Tamaño } \\
\text { muestra }\end{array}$ \\
\hline Autoridades & $\begin{array}{l}\text { Autoridades cuyos puestos estuvieran directamente relacio- } \\
\text { nados con el programa, el turismo y la economía, tanto del } \\
\text { período actual como del período pasado (presidente muni- } \\
\text { cipal, secretario de turismo, regidores de cultura y turismo, } \\
\text { tesorero, secretario de economía). }\end{array}$ & - & 10 \\
\hline Ciudadanos & $\begin{array}{l}\text { Habitantes mayores de } 18 \text { años, residentes del Pueblo } \\
\text { Mágico. }\end{array}$ & 59.125 & 361 \\
\hline Visitantes & $\begin{array}{l}\text { Turistas, ya sea locales, nacionales o extranjeros que visi- } \\
\text { tan el Pueblo Mágico. Se calculó el promedio anual; poste- } \\
\text { riormente, se calculó un promedio mensual y finalmente un } \\
\text { promedio diario, para lo cual se estableció la base promedio } \\
\text { de } 15 \text { días de afluencia entre fines de semana y festividades } \\
\text { específicas de los Pueblos Mágicos. }\end{array}$ & 382 & 189 \\
\hline Empresarios & $\begin{array}{l}\text { Encargados, dueños, gerentes de empresas y negocios rela- } \\
\text { cionados con la actividad turística (hoteles, hostales, mote- } \\
\text { les, restaurantes, fondas, tiendas de artesanías, servicios de } \\
\text { transporte, entre otros). }\end{array}$ & 120 & 91 \\
\hline
\end{tabular}

Fuente: elaboración propia a partir de información obtenida de INEGI (2019 y 2020) y SECTUR (2019). 
Previo al trabajo de campo, se desarrolló una exhaustiva búsqueda de información secundaria para obtener la base de los tamaños de muestra, así como para determinar las fechas de aplicación más adecuadas de los instrumentos de recolección de información y con ello alcanzar el objetivo.

Las encuestas se realizaron con base en un cuerpo colegiado con aporta- ciones de diversos profesores investigadores. Dichas encuestas fueron revisadas y validadas conforme a las exigencias de un estudio de esta naturaleza, verificando la confiabilidad de los instrumentos a través de la validez del contenido y del constructo. En la tabla 2 se muestra la conformación de las variables de estudio para cada una de las encuestas, de acuerdo con la población objeto de estudio.

Tabla 2. Características de los instrumentos de recopilación de datos para la investigación

\begin{tabular}{|c|c|c|c|}
\hline Población & Principales variables & $\begin{array}{c}\mathrm{N} .{ }^{\circ} \mathrm{de} \\
\text { preguntas }\end{array}$ & $\begin{array}{l}\text { Unidad de } \\
\text { medida }\end{array}$ \\
\hline Autoridad & $\begin{array}{l}\text { Principal actividad económica, turismo, actividad económica con } \\
\text { mayores ingresos, actividad económica mayor generadora de } \\
\text { empleos, inversión pública, cambios en actividades económicas } \\
\text { tradicionales debido al turismo, evaluación de la infraestructura, } \\
\text { influencia del turismo en el comportamiento de la ciudadanía, } \\
\text { trato de las autoridades del Gobierno Estatal y Federal. }\end{array}$ & 16 & $\begin{array}{c}\text { Alfanumérica } \\
\text { y numérica }\end{array}$ \\
\hline Empresario & $\begin{array}{l}\text { Giro de la empresa, tamaño de la empresa, número de traba- } \\
\text { jadores, turismo, actividades económicas que aportan mayor } \\
\text { riqueza, ingreso económico mensual de la empresa, valuación del } \\
\text { contexto económico de la comunidad a partir del nombramiento } \\
\text { como Pueblo Mágico, empresas relacionadas con el turismo que } \\
\text { se han creado, proyectos en los que se ha generado inversión } \\
\text { por parte de la empresa en los últimos tres años, beneficios con } \\
\text { que cuenta el capital humano de la empresa, forma de remune- } \\
\text { ración de los trabajadores, prestaciones recibidas por los traba- } \\
\text { jadores en la empresa, jornada laboral, tipo de local principal de } \\
\text { la empresa, modalidad de posesión del local, comportamiento de } \\
\text { ingresos en los últimos tres años, crecimiento de competencia a } \\
\text { partir del nombramiento de Pueblo Mágico, alianzas estratégicas, } \\
\text { período de mayor ingreso en el año, fuente de financiamiento } \\
\text { de la empresa, capacitación, inclusión en la empresa, ingresos } \\
\text { generadores de bienestar familiar, participación de la empresa } \\
\text { en actividades de la comunidad, mejora en calidad de vida, dis- } \\
\text { tintivos de la empresa, valuación de mejora de calidad de vida } \\
\text { de los empresarios. }\end{array}$ & 40 & $\begin{array}{l}\text { Alfanumérica } \\
\text { y numérica }\end{array}$ \\
\hline Ciudadano & $\begin{array}{l}\text { Principal actividad económica, actividad económica con mayores } \\
\text { ingresos, interés para emplearse por actividad económica, cam- } \\
\text { bios en actividades económicas tradicionales debido al turismo, } \\
\text { evaluación de situación económica de la comunidad, turismo } \\
\text { como actividad de beneficio general, inversión turística, incre- } \\
\text { mento en número de visitantes, período de mayor afluencia de } \\
\text { visitantes, respeto a tradiciones y cultura por parte de los visitan- } \\
\text { tes, los visitantes ocasionan problemas, principales recursos de la } \\
\text { comunidad, recursos más valorados por la comunidad, cambios } \\
\text { en los recursos naturales en los últimos tres años, valuación de } \\
\text { mejora de calidad de vida de los pobladores. }\end{array}$ & 24 & $\begin{array}{l}\text { Alfanumérica } \\
\text { y numérica }\end{array}$ \\
\hline
\end{tabular}




\begin{tabular}{|c|c|c|c|}
\hline Población & Principales variables & $\begin{array}{c}\text { N. }{ }^{\circ} \text { de } \\
\text { preguntas }\end{array}$ & $\begin{array}{l}\text { Unidad de } \\
\text { medida }\end{array}$ \\
\hline Turista & $\begin{array}{l}\text { Lugar de procedencia, valuación de experiencia en alojamiento, } \\
\text { valuación de experiencia en comercios, valuación de experien- } \\
\text { cia en la relación calidad/precio del destino, valuación de trans- } \\
\text { portes públicos, valuación hospitalidad/trato recibido, valuación } \\
\text { de estancia general en el destino, valuación de la información } \\
\text { turística, valuación de la oferta cultural y de ocio, valuación de } \\
\text { accesibilidad, valuación de conservación del entorno, valuación } \\
\text { de seguridad, valuación de señalización, valuación de los servi- } \\
\text { cios de restauración, valuación general de la calidad de los ser- } \\
\text { vicios, recomendación. }\end{array}$ & 63 & $\begin{array}{l}\text { Alfanumérica } \\
\text { y numérica }\end{array}$ \\
\hline
\end{tabular}

Fuente: elaboración propia (2021).

El trabajo de campo se realizó en dos olas de tiempo. La temporalidad para la aplicación de las dos olas de tiempo fue enero y febrero de 2020, contando con el apoyo de estudiantes del Centro de Estudios Tecnológicos Industrial y de Servicios n. 114 (CETis 114 - DGETI) de Jerez, Zacatecas, a quienes previamente se les capacitó para la aplicación de cada tipo de encuesta. Se armaron cuadrillas de aplicación y los integrantes del cuerpo académico de los Servicios Tecnológicos e Investigación para el Desarrollo e Innovación Organizacional supervisaron la aplicación por parte de los equipos de apoyo y también participaron en el levantamiento de la información.

Los datos recabados se procesaron y analizaron en el programa Statistical Package for the Social Sciences (SPSS) versión 23, utilizando un método de análisis descriptivo de la información, con tabulaciones cruzadas sobre variables de interés.

\section{Resultados}

\section{Autoridades}

Los resultados obtenidos reflejan que las autoridades de esta localidad coinciden en que el turismo es una actividad complementaria. De hecho, identifican la agricultura, la ganadería, la forestación y la pesca como las actividades que generan mayores ingresos económicos a la comunidad (70 $\%)$, después el comercio (20\%) y finalmente el turismo (10\%).

El $80 \%$ coincide en que la inversión pública en el sector turismo ha sido baja, ya que no supera más allá del $20 \%$ del total del presupuesto. Desde la percepción de las autoridades de esta localidad, no ha existido un abandono de las actividades económicas tradicionales debido al turismo. Aunque se han desarrollado algunas actividades asociadas al turismo, como festivales, eventos artísticos, entre otros, la población sigue manteniéndose en la misma actividad económica en la que se encontraba antes del nombramiento de Pueblo Mágico a la localidad.

La infraestructura de servicios y de instalación de nuevos comercios se aprecia con mejoras parciales (70 \%) desde que la comunidad cuenta con la marca de Pueblo Mágico, mientras que, para la pavimentación de calles y 
espacios públicos, el total de las autoridades considera que han mejorado solo parcialmente.

Desde la perspectiva de la autoridad, la apertura al turismo en la comunidad ha generado una serie de cambios parciales, principalmente en el comportamiento de la población con respecto a su cultura, costumbres y tradiciones (70\%); sin embargo, en cuanto al comportamiento de la población frente a la organización de la comunidad y la economía de la familia no se identifican cambios (60 \%) o solo de forma parcial (40 \%).

El hecho de contar con la marca de Pueblo Mágico, de acuerdo con las autoridades, no influye para que las autoridades o dependencias del gobierno ofrezcan un trato especial, ya sea respecto a proyectos especiales, a financiamiento o incluso a recursos materiales o en especie.

En general, se tiene identificado por parte de las autoridades que el desarrollo de la comunidad ha sido bueno a partir de la operación del programa; sin embargo, se resalta la importancia de asignar mayor presupuesto para garantizar la conservación de los atractivos, pero también para incrementar los productos turísticos.

\section{Ciudadanos}

La población encuestada identifica que la principal actividad económica de la comunidad es la agricultura/ganadería con un 32,1\%, seguido prácticamente en empate por el turismo y el comercio/servicios con un 29,6
$\%$ y un $29,4 \%$, respectivamente. Sin embargo, a pesar de esto, consideran que la actividad que más ingresos genera a la comunidad es el comercio/ servicios con un $34,1 \%$, seguida del turismo con un $32,7 \%$ y la agricultura/ ganadería con un 25,8 \%. Lo anterior coincide con la información económica de la localidad, por lo que queda claro que, a pesar de ser un Pueblo Mágico, la población no considera el turismo como la actividad principal ni la que genera el mayor ingreso.

En la figura 1 se ilustra el valor perceptual acerca de si el Programa Pueblos Mágicos ha contribuido en el desarrollo de la localidad desde el punto de vista de este grupo de interés.

Figura 1. Valoración del programa para el desarrollo de la localidad.

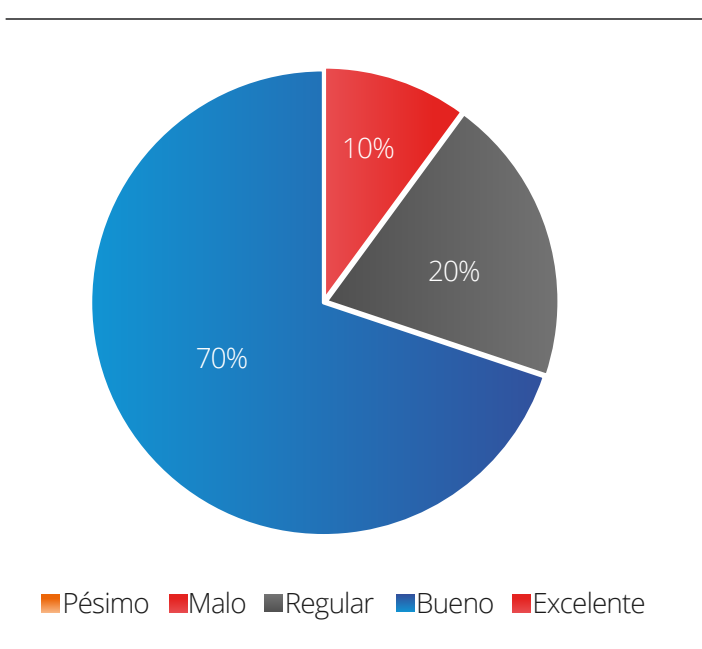

Fuente: elaboración propia (2021).

A pesar de que existe un alto interés en la comunidad por emplearse en actividades relacionadas con el turismo $(51,2 \%)$, los pobladores reconocen que aún no se ha dado un cambio de actividades económicas tradicionales por actividades del turismo (58,8 \%) 
y detectan que este trae un beneficio principalmente para los empresarios y comerciantes (51,5\%). La población jerezana encuentra que no existe un involucramiento verdadero con la comunidad por parte de las empresas turísticas, ya que rara vez o nunca $(36,6 \%)$ o solo algunas veces $(53,2 \%)$ contribuyen en actividades sociales/ culturales en la comunidad. Lo anterior es importante, pues no se debe olvidar que el Programa Pueblos Mágicos tiene como uno de sus elementos la gente, y si esta no siente el compromiso de las empresas que están explotando el lado económico del turismo, puede cambiar su postura y rechazar algunas propuestas que en un futuro las propias autoridades o empresarios pudieran plantear para atraer más turismo.

Los pobladores reconocen que luego del nombramiento como Pueblo Mágico el arribo de visitantes se ha incrementado $(78,1 \%)$ e identifican el período de enero a abril como el de mayor afluencia. Esto coincide con las principales fiestas de esta comunidad, como la feria, la Jerezada, el carnaval, las cabalgatas, entre otras, las cuales suceden durante ese período. Si bien la mayoría de los ciudadanos jerezanos considera que los turistas son respetuosos con las tradiciones, costumbres y recursos de la comunidad $(74,5 \%)$, un $25,5 \%$ considera que no lo son y que, además, ocasionan problemas en la comunidad.

En la figura 2 se ilustra la percepción que tienen los habitantes de Jerez respecto al carácter de influencia de los visitantes en la comunidad.
Figura 2. Influencia de los visitantes en la comunidad.

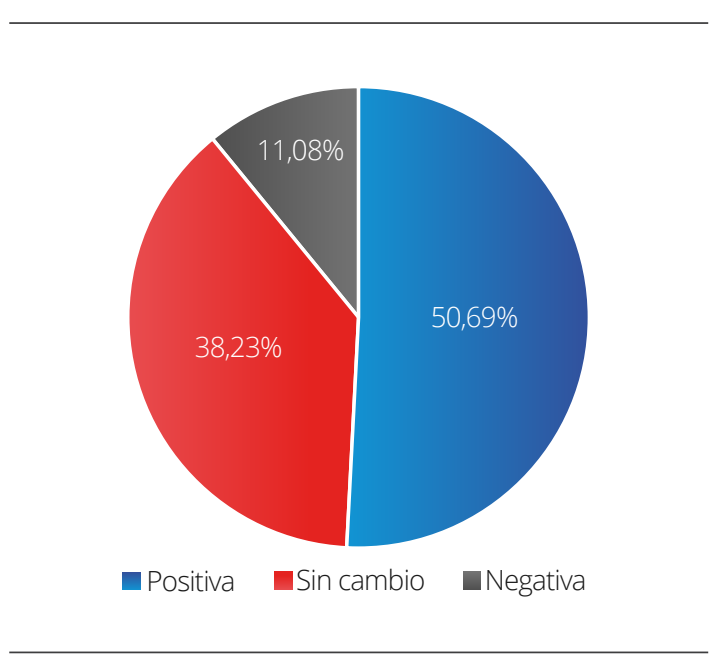

Fuente: elaboración propia (2021).

Los recursos que la población considera que son los más importantes con los que cuenta su comunidad son los monumentos históricos (74,8 \%) y los recursos forestales (14,4\%), al manifestar que la gente de la comunidad valora más estos recursos $(68,7$ $\%$ y $20,8 \%$, respectivamente).

Se percibe la existencia de cambios negativos con respecto a los recursos naturales en los últimos tres años, lo que, según manifiestan los pobladores, se debe principalmente al cambio climático (44,6 \%) o a los intereses económicos (29,1\%). El 59,6 \% de la población no identifica o tiene conocimiento de que se están emprendiendo acciones para conservar los recursos naturales. Se considera que el empleo ha crecido debido al turismo en la zona; sin embargo, este tipo de empleo es solo temporal (80,6\%), según la percepción de la población. Finalmente, se presenta la figura 3, que registra la opinión sobre si el Programa Pueblos Mágicos ha contribui- 
do a la mejora de la calidad de vida de los habitantes, lo que notoriamente se ubica en la calificación media hacia abajo.

Figura 3. Valoración de mejora en la calidad de vida de los habitantes.

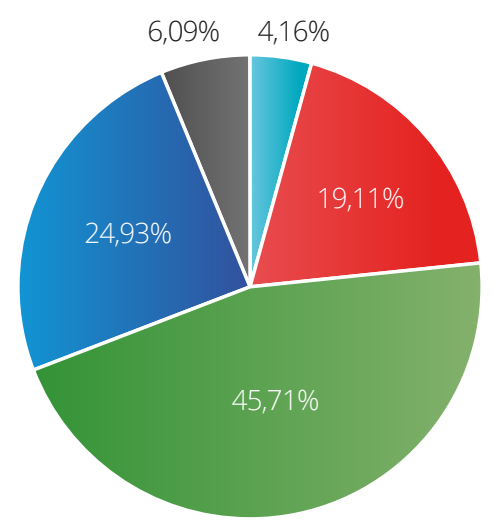

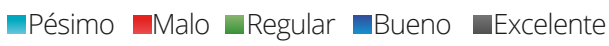

Fuente: elaboración propia (2021).

\section{Empresarios}

Los empresarios aportaron información valiosa, como el hecho de que, desde su punto de vista, en Jerez, el turismo lo observan mayormente como una actividad complementaria, lo cual es coincidente en casi todos los niveles escolares, exceptuando en aquellos empresarios que únicamente cuentan con la primaria, cuya opinión es que el turismo sí es una actividad principal. En este grupo objeto de estudio, el 59,3\% considera que debido al Programa Pueblos Mágicos ha crecido la competencia. Con respecto a la posesión del local de la empresa, el $50,5 \%$ renta el local y un 39,6 \% tiene local propio; predominan los locales independientes con un $78 \%$. Al solicitarles señalar qué empresas se han creado a partir de que se obtuviera el nombramiento de Pueblo Mágico, se obtuvo que un 41,8 \% corresponde a empresarios locales que abren negocios, el $22 \%$ considera que no se han creado empresas, el 18,7\% se ubica entre las franquicias y el 17,6 \% representa las empresas foráneas.

En la figura 4 se aprecia la percepción que tienen los empresarios acerca de si la actividad turística en el Pueblo Mágico de Jerez es una actividad principal o complementaria, en contraste con el nivel educativo del empresario encuestado.

Figura 4. Percepción de la actividad turística con base en el nivel educativo del empresario encuestado.

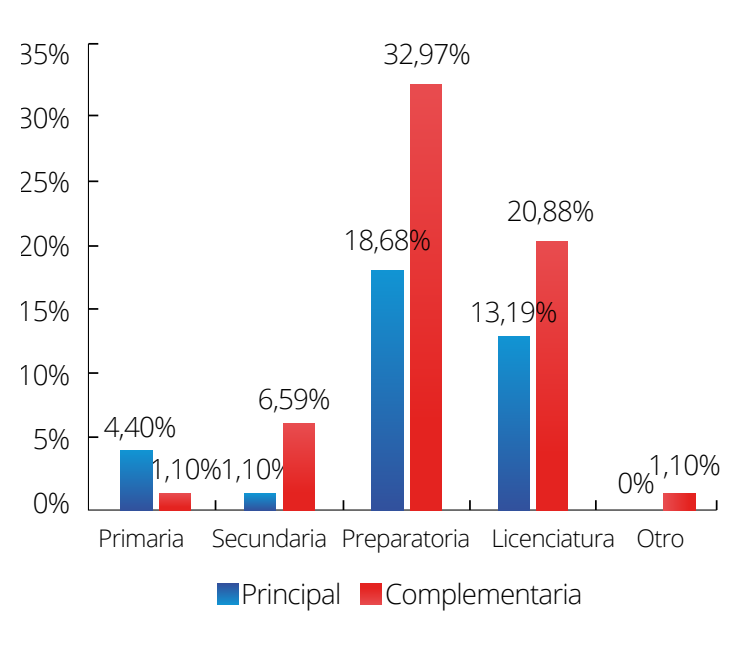

Fuente: elaboración propia (2021).

El 40,7 \% de los empresarios señala que el turismo y los servicios turísticos son las actividades económicas que aportan mayor riqueza. En segundo lugar, ubican lo agropecuario con el $31,9 \%$ y en tercer lugar el comercio con el 23,1 \%. Es interesante detectar que solo el 49,5\% de los empresarios 
considera que, desde que se otorgó el nombramiento de Pueblo Mágico, la situación económica de la comunidad ha propiciado más ingresos, mientras que el 30,8\% manifiesta que el nombramiento no ha influido, pues siguen igual y el 19,8\% plantea que, incluso, se han tenido pérdidas. Al indagar acerca de los períodos en los que se reciben mayores ingresos en las empresas, los resultados quedan reflejados en la figura 5, la cual muestra que el de mayor porcentaje coincide con las festividades que más atraen visitantes, como la Feria de la primavera, el carnaval, la Jerezada, la cabalgata, la Quema de Judas, entre otras.

En la figura 6 se ilustra la valoración percibida sobre los ingresos de la em- presa en los últimos tres años, con base en el giro de esta.

Figura 5. Período con mayor ingreso económico para las empresas de la localidad.

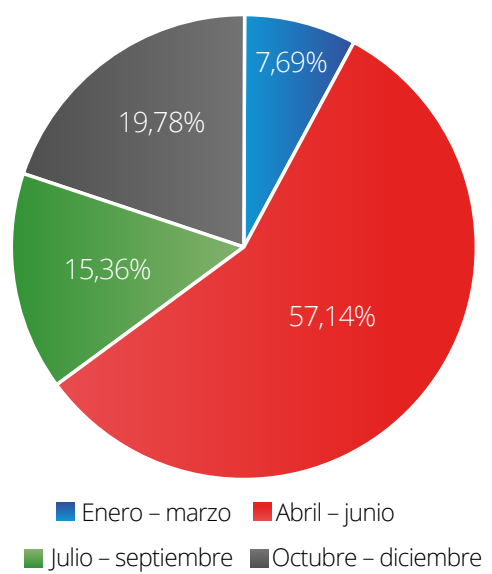

Fuente: elaboración propia (2021).

Figura 6. Valoración de los ingresos para la empresa en los últimos tres años.

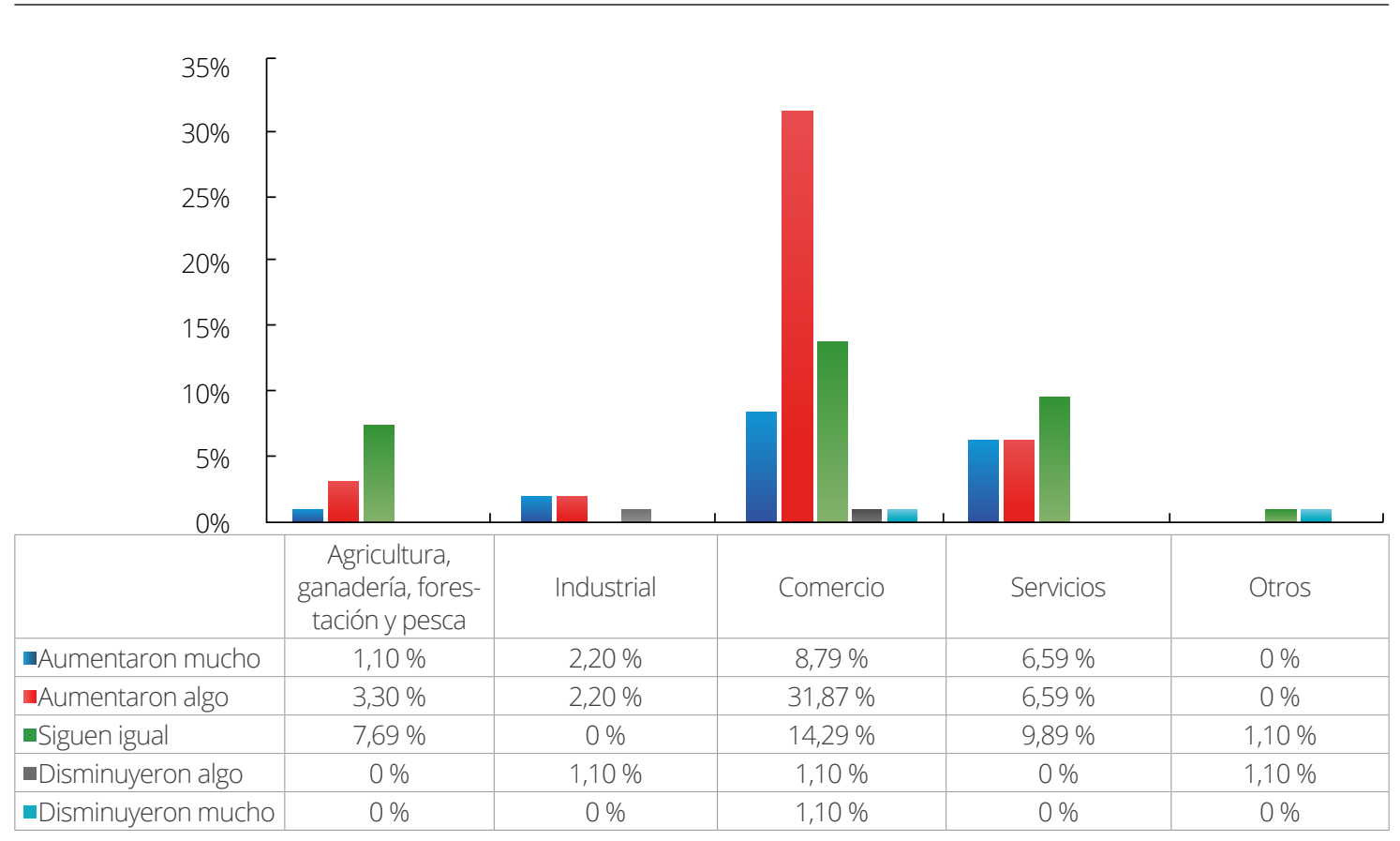

Fuente: elaboración propia (2021). 
También consideran que la calidad de vida de los niños, mujeres y ancianos de la comunidad no ha mejorado con el turismo (68,1\%). Lamentablemente, se evidencia también que la mayoría de los empleos que prevalecen en las empresas son remunerados principalmente con el salario mínimo $(45,1 \%)$ o según el número de días trabajados (36,3 \%) y solo un $18,7 \%$ de las empresas otorga un salario de acuerdo con el mercado. Incluso, la principal prestación ofrecida a los empleados son los días de descanso $(42,9 \%)$. El $53,8 \%$ no cuenta con prestaciones sociales, como servicio médico, vacaciones pagadas, reparto de utilidades o fondo de retiro. Las jornadas laborales son mayormente matutinas (45,1\%) o mixtas (30,8 \%).

Finalmente, en la figura 7 se puede identificar la valoración que dan los empresarios acerca de si el Programa de Pueblos Mágicos ha mejorado la calidad de vida de los empresarios.

Figura 7. Valoración de mejora en la calidad de vida desde el punto de vista de los empresarios.

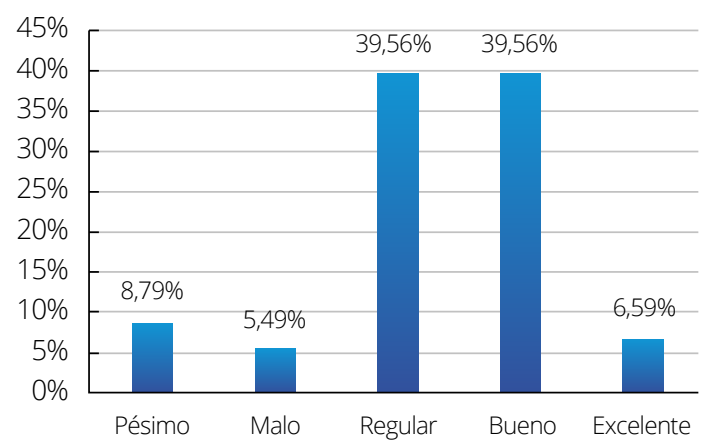

Fuente: elaboración propia (2021).

\section{Visitantes}

La mayoría de los turistas que visitan el Pueblo Mágico de Jerez de García Salinas son originarios del interior de la república e incluso del propio estado de Zacatecas, según cifras ya documentadas por las autoridades estatales de turismo y que en el presente estudio se comprueban, puesto que, aun cuando se aplicó el instrumento en dos olas de tiempo, un $93,1 \%$ de los visitantes era del interior del país. Únicamente el 64,6\% utiliza el servicio de hotel como alojamiento, mientras que un $15 \%$ se aloja en pensiones y un $10 \%$ lo hace en casa de huéspedes; un 10,4 \% de los turistas no requieren el servicio, dado que este lo adquieren en la capital del estado, que está relativamente cerca, por lo que solo van, visitan, hacen sus recorridos de interés y regresan a su destino principal. Dentro de las experiencias de los turistas, los resultados nos arrojan que los productos que suelen adquirir durante su visita son principalmente productos gastronómicos locales $(77,2$ \% correspondiente a salsas, semillas, nieves, ates, entre otros), mientras que las artesanías apenas alcanzan un 12,7 \%.

La percepción en cuanto a la relación de la calidad con respecto al precio del destino tiene sus particularidades; por ejemplo, en cuanto a si es accesible, solo un 27,5\% tiene calificaciones positivas, mientras que el resto considera que no lo es. Por otro lado, la valoración de las formas de pago fue positiva, pues para quienes requirieron ese servicio resultó una experiencia favorable $(56,7 \%)$. 
En particular, esta comunidad tiene mucho que mejorar para poder proporcionar una mejor experiencia al turista en materia de transportes públicos, ya que, tanto para los autobuses como para el transporte urbano o taxis, las valoraciones de experiencia no se inclinan hacia lo positivo, lo cual se debe al costo, a las condiciones de las unidades o incluso a la disponibilidad de horarios de dichos servicios.

Otro de los aspectos que sorprende entre los resultados tiene que ver con las experiencias en cuanto a la hospitalidad y la estancia en sí en la comunidad, pues lamentablemente se tiene un área enorme de oportunidad al tener un $61,9 \%$ y un $75,6 \%$ de valoraciones no positivas, respectivamente. Es importante hacer algo al respecto para mejorar la experiencia de los visitantes, ya que en la medida en que estos se sientan como en casa es que pueden alargar su estancia o llegar a recomendar la visita a amigos y familiares. En la figura 8 puede apreciarse cómo valoraron los visitantes su experiencia en cuanto a los servicios de hospedaje. Predominaron las valoraciones regular a pésimo en cuanto a hotel y pensión mientras que las casas de huéspedes fueron valoradas principalmente como excelente $y$, en segunda instancia, malo. Solo un 44,4 $\%$ de los visitantes valoró positivamente el cumplimiento de sus expectativas con respecto al Pueblo Mágico.

Figura 8. Valoración de la experiencia en los servicios de hospedaje y alojamiento en la localidad, por parte de los visitantes.

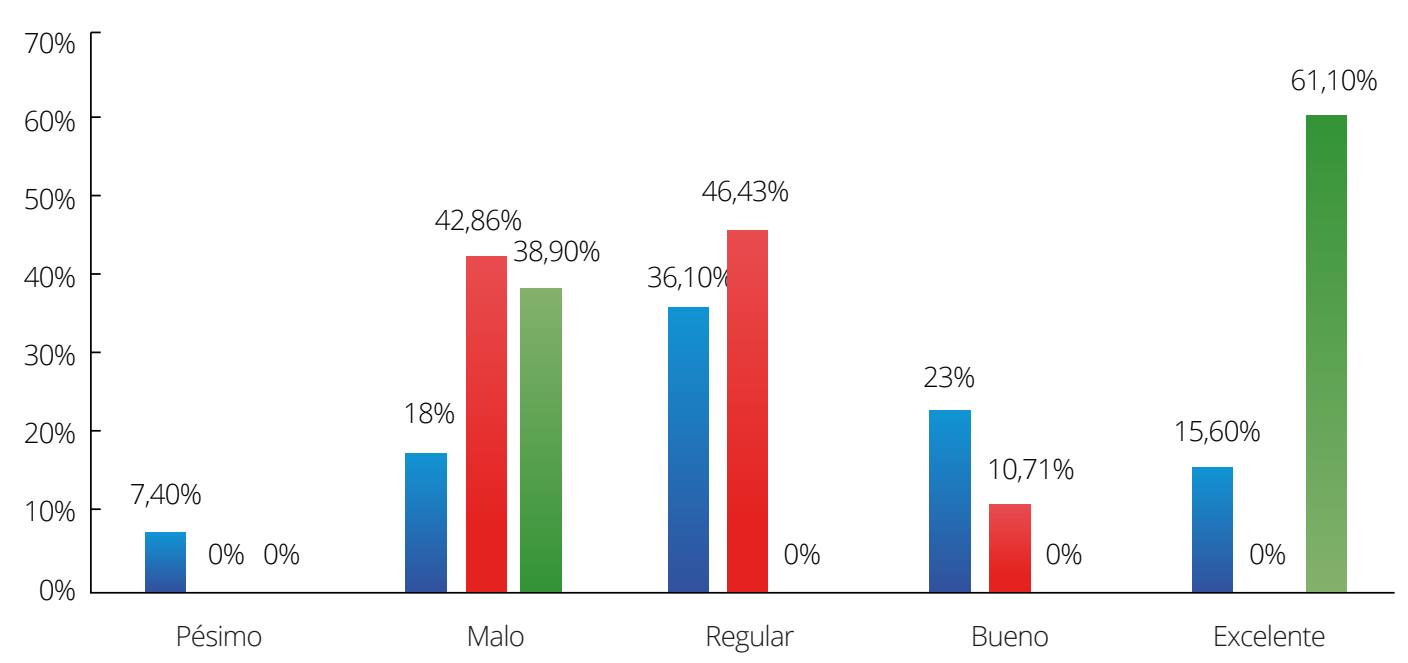

- Hotel a Pensión - Casa de huéspedes

Fuente: elaboración propia (2021). 


\section{Discusión}

Aunque el programa Pueblos Mágicos del Gobierno Federal intenta impulsar el desarrollo de los pueblos, como se menciona en el presente estudio, de acuerdo con la percepción de los pobladores, empresarios y autoridades, no existe información suficiente para asegurar que esto ha sucedido en el Pueblo Mágico de Jerez, en el estado de Zacatecas. Por el contrario, sus percepciones evidencian que no existe articulación entre el gobierno, las empresas y la propia comunidad. Las pequeñas y medianas empresas que se encuentran dentro de estos pueblos no están siendo incluidas y por ello tampoco se involucran en las actividades de la comunidad, por lo que los habitantes las sienten ajenas, situación que no contribuye a un escenario de ganar-ganar, ya que en la medida en que se sientan acompañados e integrados en este escenario los resultados terminarán beneficiando al turista, que apreciará los cambios desde la actitud y hospitalidad de la gente, hasta la mejora en los comercios y servicios.

Esta investigación nos arroja que aún existen áreas de oportunidad en cuanto a diversos temas con respecto a este Pueblo Mágico, como infraestructura turística, carreteras, señalética, entre otros, de acuerdo con lo percibido por las autoridades, los propios turistas, los habitantes y los empresarios de Jerez. Esto coincide con el diagnóstico y la evaluación del Programa Pueblos Mágicos que realizó SECTUR en 2013, entre cuyas conclusiones señaló que estos tuvieron la calificación más baja con respecto a satisfacción turística, debido principalmente a la falta de infraestructura y de servicios generales y turísticos (SECTUR, 2014).

En esta investigación colaborativa se evaluó el impacto de la aplicación del Programa Pueblos Mágicos, el cual no es muy alentador en la localidad de Jerez de García Salinas, ya que a pesar de que sí se generan empleos, estos son en su mayoría de carácter temporal y con pocas o nulas prestaciones, según las percepciones de los diferentes públicos investigados. Aun cuando en investigaciones anteriores en otros Pueblos Mágicos se hayan obtenido resultados diferentes, por ejemplo, la investigación de Velarde et al. (2009), que concluye que en las localidades abatidas por la pobreza, cuyos pobladores emigraban en busca de mejores oportunidades de vida, ahora es evidente la reactivación de las actividades productivas y la generación de empleo, como en el caso de Sinaloa. En las figuras 9 y 10 se ilustran datos relevantes acerca de hoteles y su comportamiento a través del tiempo. Adicionalmente, se incrementó el número de moteles y centros de esparcimiento (bares, discotecas o similares), aunque no de forma significativa, ya que en 2019 solo existían dos de cada categoría (SECTUR, 2021).

El número de unidades económicas se ha visto favorecido después de contar con la marca Pueblos Mágicos; sin embargo, la actividad turística (entendida como toda actividad relacionada con la entrada, recepción, alojamiento, esparcimiento, alimenta- 
ción o actividad artesanal basada en el turismo) no figura como una actividad principal para las autoridades, la sociedad jerezana, ni los propios empresarios, como se evidencia en la presente investigación. Las oportunidades que pueden presentarse son aprovechadas en gran medida por personas externas a la comunidad. De acuerdo con los comentarios de algunos de los encuestados, pareciera que el gobierno municipal les da prioridad a los foráneos.

Figura 9. Hoteles con otros servicios integrados y su comportamiento a través de los años en Jerez, Zacatecas.

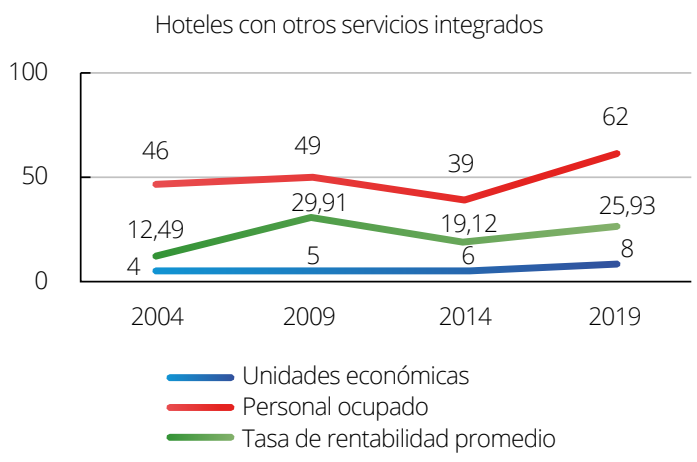

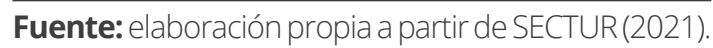

Figura 10. Hoteles sin otros servicios integrados y su comportamiento a través de los años en Jerez, Zacatecas.

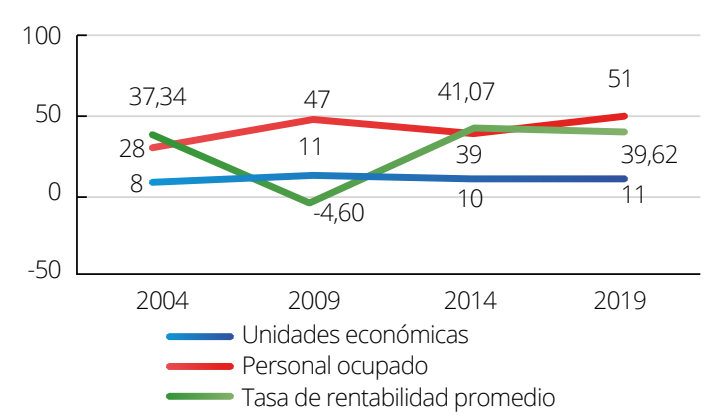

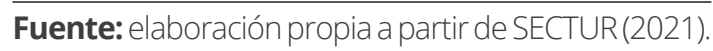

Es importante señalar que, para los visitantes, el hecho de no haber tenido acceso a una oferta cultural y de ocio amplia, la carencia de información turística disponible sin costo, la señalización y la seguridad (un tema que cobra importancia en la actualidad en cualquier destino y que representa un reto significativo que tiene que abordarse para poder brindar certidumbre a los visitantes) ha implicado que no recurran a pernoctar $y$, por ende, no utilicen los servicios de hospedaje, sino que el lugar solo se vea como un espacio de tránsito o visita rápida. Todo esto invita a valorar la utilización de estrategias digitales y de la innovación tecnológica que permitan hacer frente a las necesidades actuales del turismo, sea de carácter local, nacional o internacional, para mostrarles a los visitantes la riqueza cultural de este Pueblo Mágico.

Autores como Caro et al. (2015) proponen que, debido a la globalización, es importante atrapar a través de elementos de identidad de una región a quienes están interesados en sus cualidades y en recibir algo más para tomar decisiones con respecto a los sitios a visitar. Este planteamiento coincide con el de Shaadi et al. (2017), quienes señalan que los turistas que visitan estas localidades esperan satisfacer sus expectativas y necesidades. Además, derivado de los avances en el uso de la tecnología y comunicaciones, los visitantes reflejan una necesidad de espacios turísticos en los que se encuentren bien articulados y en existencia suficiente los atractivos y servicios turísticos. Por lo anterior, un trabajo coordinado entre el ayun- 
tamiento y el Comité Ciudadano del Pueblo Mágico, sin duda, podría contribuir enormemente a aligerar la brecha que se logra identificar entre estos elementos indispensables para el desarrollo: la sociedad, la empresa y la autoridad. Este colectivo de investigación comparte totalmente lo planteado por Porter y Kramer con respecto a que las empresas y la sociedad deben unir sus esfuerzos para obtener un beneficio común, lo que han denominado valor compartido, es decir, una nueva manera de lograr el éxito económico (Porter \& Kramer, 2011).

El seguimiento adecuado e integral por parte de las autoridades competentes es algo en lo que se debe seguir trabajando, pues no se trata solo de aprobar el recurso, sino de verificar su correcta aplicación y medir constantemente el impacto que se ha tenido con ello, para, en caso necesario, poder reorientar esfuerzos y proyectar adecuadamente el desarrollo turístico de la localidad, sin que esto derive en la sobreexplotación de recursos naturales o en la deformación de la riqueza cultural-social que aporta esta localidad y, como afirman Núnez y Ettinger (2020), sin descuidar el darle el continuo impulso a las actividades propias de la localidad, esto debido a que el Programa Pueblos Mágicos busca fomentar el turismo, pero como parte sustentable de las actividades ya desarrolladas localmente. Esta situación también fue resaltada por López (2018), quien manifiesta que el turismo debe integrarse en un sistema donde se complemente con otros sectores, buscando el beneficio de la comuni- dad y no solo de algunos grupos sociales, tal como se plantea en uno de los objetivos de la Estrategia Nacional de Pueblos Mágicos: fomentar el desarrollo justo y equilibrado entre los individuos, comunidades y regiones para democratizar los beneficios del turismo en las comunidades receptoras (SECTUR, 2020). Por lo anterior, no se debe dejar de lado la oportunidad de cohesionar al motor económico de la localidad, el turismo.

No se debe dejar de lado uno de los elementos principales de esta localidad: su gente, los habitantes de este Pueblo Mágico. Considerar lo que sienten y piensan con respecto a lo que ha implicado la implementación de este programa en la localidad es importante, lo cual coincide con lo manifestado por Rojo et al. (2017) en el sentido de que se dejan de lado aspectos como los efectos del turismo en el espacio (patrimonio natural y construido) y en el habitante tradicional. Se considera que, si la población se siente orgullosa de que su comunidad tenga la distinción de Pueblo Mágico, esto permitirá ir alcanzando la consolidación del programa, dejando de lado que solo sea interpretado como un programa de buenas intenciones, según Álvarez (2017).

Consideramos que los resultados arrojados en esta investigación se ven cobijados por la nueva visión de la SECTUR, planteada en la Estrategia Nacional de Pueblos Mágicos, en la que consideran que el turismo se transforma en una herramienta de reconciliación social y, así mismo, se asume el reto de impulsar un desarro- 
Ilo turístico más equilibrado, ampliar la infraestructura y los servicios, así como fomentar la integración y la diversificación del turismo desde el ámbito local y regional (SECTUR, 2020).

\section{Conclusiones}

Se sugiere que a partir de esta investigación se dé la pauta para que se aplique una estructura renovada dentro del programa, la cual permita entender el desarrollo de las actividades que se requieren llevar a cabo desde el principio para postularse como Pueblo Mágico, y aquellas que sin ser menos importantes apoyan el desarrollo de este para su permanencia dentro de la marca Pueblo Mágico.

Esto nos lleva a sugerir la incursión en el trabajo de un modelo con enfoque de quíntuple hélice, en el que Gobierno, universidad, medio ambiente, sociedad y empresa confluyan para provocar una movilidad económica y de recurso humano, favoreciendo con ello la innovación de esta comunidad en turismo, partiendo de proyectos basados en arropar la hélice del medio ambiente, de tal modo que se garantice que no se descuide de ninguna manera la vasta bondad natural prevaleciente en la zona, así como el desarrollo turístico, sino que de forma adecuada se proyecten los alcances y se optimicen los esfuerzos. Lo anterior cobra fuerza, ya que, en el marco del Plan Municipal de Desarrollo 2019-2021, el Ayuntamiento de Jerez (2019) ha considerado como parte de su tercer eje transversal, como una de sus líneas estratégicas, el turismo sostenible, cuyo objetivo radicó en el mejoramiento de todos los servicios turísticos para contribuir a la estadía del turista en Jerez; sin embargo, las estrategias no reflejan un enfoque sostenible.

En la investigación realizada en el Pueblo Mágico de Loreto en Baja California Sur, Olmos et al. (2021) concluyen que, adicional al aprovechamiento de los atractivos históricos y culturales, las actividades recreativas y de esparcimiento también se relacionan con el uso de los recursos naturales, por lo que ofertan actividades como senderismo, tirolesa, fotografía, cabalgata, observación de flora y fauna, ciclismo, entre otras. Durante la investigación pudimos observar los múltiples espacios naturales que forman parte del Pueblo Mágico de Jerez, los cuales son orgullo de sus pobladores y no están contemplados como actividades turísticas de la región, por lo que consideramos que pueden ser explotados para generar flujo de turismo, ingresos y trabajo para los lugareños a través de estrategias turísticas adecuadas que fortalezcan el desarrollo sustentable de este Pueblo Mágico, sin olvidar lo señalado por Gauna (2019) acerca de considerar los impactos al patrimonio tanto cultural como natural, planteamiento que exige el desarrollo de acciones encaminadas a su conservación y protección a lo largo del tiempo.

La estrategia digital y de innovación tecnológica es una de las áreas de oportunidad para este Pueblo Mágico, dado que pueden identificarse opciones como la difusión en redes sociales, la utilización de kioscos electrónicos, 
las adecuaciones de infraestructura y señalética para el turismo de inclusión (discapacidad visual, auditiva y motriz), entre muchas otras que resulten atractivas para los visitantes.

Finalmente, queremos plantear que el pensar a futuro y poder tender los hilos para generar un subclúster turístico puede ser una tarea importante que beneficie no solo a este Pueblo Mágico, sino a otros del estado e incluso de la región.

\section{Información complementaria}

Agradecimientos a la Red de Administración y Negocios por la invitación a participar en la investigación sobre Pueblos Mágicos. A los directivos y alumnos del CETis 114 (DGETI) de Jerez, Zacatecas, a los directivos de la Universidad Tecnológica del Estado de Zacatecas y a las autoridades del municipio de Jerez por su colaboración para contestar la encuesta y a la Red GESTIO por generar la apertura para la generación y divulgación de investigaciones como esta.

Contribuciones de autoría: todas y cada una de las autoras contribuyeron notablemente con la realización de la investigación y la redacción del artículo.

Conflictos de interés: declaramos que no existe alguna relación, condición, circunstancia o posición que pueda afectar la objetividad del presente artículo.

Financiamiento: la investigación fue autofinanciada por el cuerpo académico UTEZAC-08.

\section{Referencias}

Álvarez-Quijada, R. (2017). Percepción ciudadana sobre el programa de Pueblos Mágicos. Estudio estadístico en Álamos, Sonora. En Enríquez, J. et al., Patrimonio y Turismo. Un acercamiento a los lugares turísticos de México (p. 69). https://bit.ly/3kOBGBv

Ayuntamiento de Jerez. (2019). Plan Municipal de Desarrollo 2019-2021 de Jerez, Zac. https://bit.ly/3cnMpON

Caro-Maldonado, Y. A., Cuervo-García, E. A., \& Gómez-Camargo, A. C. (2015). Qué es y cómo se desarrolla un plan de marketing territorial. Revista GEON (Gestión, Organizaciones Y Negocios), 2(2), 76-82. https://doi. org/10.22579/23463910.102

Gauna-Ruiz de León, C. (2019). Análisis sociodemográfico, económico y turístico de los "Pueblos Mágicos" de Jalisco, México. El periplo sustentable, (36), 34-66. https://bit.ly/3CzQt98

Hernández-Sampieri, R., Fernández-Collado, C. \& Baptista-Lucio, M. (2010). Metodología de la investigación. México D.F. McGraw-Hill.

Instituto Nacional de Estadística y Geografía [INEGI] (2019). Directorio Estadístico Nacional de Unidades Económicas [DENUE]. https://bit.ly/3x0kGwU

Instituto Nacional de Estadística y Geografía [INEGI] (2020). Banco de Información Económica [BIE]. http://www3. inegi.org. $\mathrm{mx} /$ sistemas/bie

Instituto Nacional de Estadística y Geografía [INEGI] (2020). México en Cifras. Banco de Información del INEGI. http:// www3.inegi.org. mx/sistemas/mexicocifras/ 
Landeros-Villalva, H., Gomora-Serrano, M., \& Castañeda-Martínez, T. (2015). Enfoques de Análisis en Pueblos Mágicos: ¿eficacia o eficiencia? $20^{\circ}$ Encuentro Nacional sobre Desarrollo Regional en México. Cuernavaca, Morelos del 17 al 20 de noviembre de 2015. AMECIDER - CRIM, UNAM. https://bit. ly/3kRsWuh

Lopez-Levi, L. (2018). Las territorialidades del turismo: el caso de los Pueblos Mágicos en México. Ateliê Geográfico, 12(1), 6-24. https://doi. org/10.5216/ag.v12i1.45803

Núñez-Camarena, G. M. \& Ettinger-Mc Enulty, C. (2020). La transformación de un territorio cultural. El desarrollo de los Pueblos Mágicos en México: Pátzcuaro como caso de estudio. Urbano, 23(41), 40-57. https://dx.doi.or g/10.22320/07183607.2020.23.41.03

Olmos-Martínez, E., Ibarra-Michel, J. P. \& Cruz-Coria, E. (2021). Satisfacción del visitante en Pueblos Mágicos del noroeste mexicano: una mirada a Loreto, B.C.S. Gran Tour: Revista de Investigaciones Turísticas, 23(1), 3-24. https:// bit.ly/3HCzIDg

Porter M. E. \& Kramer M. (2011). La creación de valor compartido. Harvard Business Review América Latina; 1-18. https://bit.ly/3qQWyvy

Rojo-Quintero, S., Rodríguez-González, S. C. \& Castañeda-Corral, M. E. (2017) Del espacio vivido a los lugares de encuentro turístico: El Rosario Pueblo Mágico. En Enríquez, J. et al., Patrimonio y Turismo. Un acercamiento a los lugares turísticos de México (p. 51). https://bit.ly/3cubJmd

Secretaría de Turismo del Gobierno Federal [SECTUR] (2014). Evaluación de desempeño de los destinos turísticos en el marco de los convenios de coordinación en materia de reasignación de recursos (CCRR) Análisis del desempeño turístico local, Modelo de Satisfacción de los turistas. https://bit.ly/3qW6eVX

Secretaría de Turismo del Gobierno Federal [SECTUR] (2019). DATATUR, Estadísticas del Sector Turismo. Análisis Integral del Turismo. http://www.datatur. sectur.gob.mx

Secretaría de Turismo del Gobierno Federal [SECTUR] (2019). Programa Pueblos Mágicos. http://www.sectur.gob. $\mathrm{mx/gobmx/pueblos-magicos/}$

Secretaría de Turismo del Gobierno Federal [SECTUR] (2020). Diagnóstico Socioeconómico de los Pueblos Mágicos. https://bit.ly/3ctuyWt

Secretaría de Turismo del Gobierno Federal [SECTUR] (2020). Estrategia Nacional de Pueblos Mágicos. https://bit. ly/3CtoN5N

Secretaría de Turismo del Gobierno Federal [SECTUR] (2021). Compendio estadístico del turismo en México 2020. https://bit.ly/3nsdRBg

Shaadi-Rodríguez, R. M. A., Pulido-Fernández, J. I. \& Rodríguez-Herrera, I. M. (2017). El producto turístico en los Pueblos Mágicos de México. Un análisis crítico de sus componentes. Revista de Estudios Regionales, (108), 125-163. https://www.redalyc.org/articulo. oa?id=75551422005

Velarde, M., Maldonado, A. \& Maldonado M. (2009). Pueblos Mágicos. Estrategia para el desarrollo turístico sustentable: caso Sinaloa. Teoría y Praxis. 6, 81-95. https://bit.ly/30JPCWD 